\title{
Neuroprotective effect of interleukin-6 in a rat model of cerebral ischemia
}

\author{
QILIN FENG $^{1}$, YI WANG ${ }^{1}$ and YINGDA YANG ${ }^{2}$ \\ ${ }^{1}$ Department of Pharmacy, Central Hospital of Wuhan; ${ }^{2}$ School of Pharmacy, Tongji Medical College, \\ Huazhong University of Science and Technology, Wuhan, Hubei 430030, P.R. China
}

Received May 25, 2014; Accepted March 4, 2015

DOI: $10.3892 /$ etm.2015.2363

\begin{abstract}
Interleukin (IL)-6 is known to be a key cytokine in immune regulation in addition to serving crucial functions in various autoimmune diseases; however, the neuroprotective potential of IL- 6 has not been fully investigated. The aim of the present study was to investigate the neuroprotective effects of the inflammatory cytokine IL- 6 in a rat model of cerebral ischemia. Rat cerebral ischemia was induced by intraluminal middle cerebral artery occlusion. Following treatment with 500 or $50 \mathrm{ng}$ IL-6, the infarct volumes and symptoms of neurological deficit were ameliorated. Furthermore, terminal deoxynucleotidyl transferase-mediated dUTP nick end labeling staining suggested that the IL- 6 treatment reduced neuronal apoptosis in vivo, which was consistent with a lower percentage of annexin V- and caspase-3-positive cortical neurons. In addition, IL- 6 in vitro induced the phosphorylation of signal transducer and activator of transcription (STAT) 3 and the expression of induced myeloid leukemia cell differentiation protein Mcl-1, but not the expression of B-cell lymphoma 2, suggesting the activation of the Janus kinase/STAT pathway by IL-6. IL-6 also appeared to be involved in the regulation of cytokine secretion and blood-brain barrier (BBB) integrity in cerebral ischemia. IL- 6 downregulated a number of inflammatory cytokines, including tumor necrosis factor- $\alpha$ and IL-1 $\beta$, as well as myeloperoxidase activity, indicating the accumulation of granulocytes in the ischemic brain tissue IL-6 was also observed to support the integrity of the BBB by reducing Evans blue leakage in vivo and suppressing the expression of matrix metalloproteinase-9 in ischemic brain tissue. In conclusion, the results of the present study indicate that the neuroprotective effects of IL-6 in cerebral ischemia are the result of a range of processes, including the modulation of cell apoptosis, cytokine secretion and the integrity of the
\end{abstract}

Correspondence to: Dr Qilin Feng, Department of Pharmacy, Central Hospital of Wuhan, Bo'ai Building No. 2, 26 Shengli Street, Wuhan, Hubei 430030, P.R. China

E-mail: fqlwh@126.com

Key words: interleukin-6, cerebral ischemia, neuroprotection
BBB. IL-6 could therefore be used as a therapeutic agent in clinical practice.

\section{Introduction}

A previous study demonstrated that immune-related cytokines are involved in the pathology of cerebral ischemia and subsequent neuronal death (1); however, few cytokines, such as interleukin (IL)-1, nerve growth factor, transforming growth factor- $\beta$ and tumor necrosis factor (TNF)- $\alpha$, have been directly associated with cellular damage $(2,3)$ following experimentally induced cerebral ischemia. IL-6 was initially identified as B-cell stimulating factor (4), and is also synthesized by neurons and glia. IL- 6 mRNA expression in the brain is known to increase in various central nervous system (CNS) disorders, including cerebral ischemia $(5,6)$. IL-6 has been demonstrated to be crucial for neuron survival in culture $(7,8)$, and serves a key function in the regeneration of peripheral nerve cells $(9,10)$.

IL-6 functions via two subunits of its receptor: The $\alpha$-chain is the IL- 6 binding protein gp80, and the $\beta$-chain is the signal-transducing protein gp130 (11). Two pathways are activated by gp130. The first pathway, the mitogen-activated protein kinase pathway, is Ras-dependent and leads to the activation of a variety of transcription factors, such as nuclear factor for IL-6, ETS domain-containing protein Elk-1 and activator protein 1; the second is the Janus kinase (JAK)-signal transducer and activator of transcription (STAT) pathway, which involves the activation of JAK and the STAT family members STAT1 and STAT3 $(12,13)$. The two pathways have been implicated in cell proliferation and survival. Generally, the apoptosis-related B-cell lymphoma 2 (Bcl-2) protein family is believed to be a regulator of cell survival (14) and Bcl-2, which is highly expressed in malignant plasma cells, has been extensively studied among the Bcl-2 family proteins. Furthermore, a prior study has indicated that $\mathrm{Bcl}-2$ protein is able to mediate cell cycle function (15). By contrast, Bcl-xL is thought to be a potential marker of chemoresistance regulating cell apoptosis in myeloma (16). A number of studies have indicated that induced myeloid leukemia cell differentiation protein Mcl-1 is crucial for the survival of B cells, particularly during the late stages of B-cell differentiation $(17,18)$. In addition, IL-6 is known to regulate Mcl-1 and Bcl-xL proteins in myeloma cells $(19,20)$. The aim of the present study, therefore, 
is to investigate the neuroprotective effects of the inflammatory cytokine IL-6 in a rat model of cerebral ischemia, and to investigate the involvement of the JAK/STAT pathway, i.e. the phosphorylation of STAT3 following IL-6 treatment, in this process.

\section{Materials and methods}

Rat models. All experimental protocols were approved by the Institutional Animal Care and Use Committee of Tongji Medical College, Huazhong University of Science and Technology (Wuhan, China). Adult male Sprague Dawley rats weighing 250-280 g were obtained from Vital River Laboratory Animal Technology Co., Ltd. (Beijing, China). Focal cerebral ischemia was induced via intraluminal middle cerebral artery occlusion (MCAO), as described by Longa et al (21), with certain modifications. Briefly, the rats were intraperitoneally (i.p.) anesthetized with chloral hydrate (350 mg/kg), and a surgical nylon monofilament tip coated with $0.01 \%$ poly-L-lysine was then introduced into the left internal carotid artery through the external carotid stump. This filament was advanced $18-20 \mathrm{~mm}$ beyond the carotid bifurcation until a slight resistance was detected. At this point, the origin of the middle cerebral artery was obstructed by the intraluminal filament, and all blood flow from the internal carotid, anterior cerebral and posterior cerebral arteries was occluded. The body temperature of the rats was maintained at $37 \pm 0.5^{\circ} \mathrm{C}$ throughout the procedure. The filament was left in position for $2 \mathrm{~h}$ and then withdrawn. The rats were returned to their cages and closely monitored until they were observed to have recovered from the anesthesia. Any rats that exhibited an absence of neurological deficits immediately following reperfusion (neurological score, $<3$ ) were excluded from the study. Sham-operated rats were treated identically, with the exception that the MCAs were not occluded following the neck incision.

Drug preparation and treatment schedule. Recombined IL-6 was purchased from PeproTech, Inc. (Rocky Hill, NJ, USA) and dissolved in physiological saline. A total of 52 rats were divided at random into sham, saline, IL-6 (50 ng, i.p.) and IL-6 (500 ng, i.p.) treatment groups. IL-6 solution or a vehicle of physiological saline was administered $10 \mathrm{~min}$ after the MCAO procedure.

Infarct volume determination. Each rat was sacrificed $24 \mathrm{~h}$ after reperfusion and the brain was removed rapidly and frozen at $-20^{\circ} \mathrm{C}$ for $5 \mathrm{~min}$. Coronal slices were collected at points $2 \mathrm{~mm}$ from the frontal tips and immersed in $2 \%$ 2,3,5-tripenyltetrazolium chloride stain at $37^{\circ} \mathrm{C}$ for $20 \mathrm{~min}$. Following staining, color images of the slices were captured using a Kodak 7230 digital camera (Kodak, Rochester, NY, USA) and Adobe Photoshop software, version 7.0 (Adobe Systems, Inc., San Jose, CA, USA). The infarct volume was calculated using the Mias-2000 image analysis system (Institute of Graphics and Images, Sichuan University, Chengdu, China).

Neurological deficit determination. Symptoms of neurological deficit in the vehicle- and drug-treated groups were assessed after $24 \mathrm{~h}$ of reperfusion according to the method described by Longa et al (21). Neurological findings were scored on a five-point scale, as follows: No neurological deficit, 0; failure to extend right paw fully, 1; circling to the right, 2; falling to the right, 3; and inability to walk spontaneously with depressed levels of consciousness, 4 .

Terminal deoxynucleotidyl transferase-mediated dUTP nick end labeling (TUNEL) staining. To detect neuronal apoptosis, in situ nick end labeling was performed using a commercial kit (In Situ Apoptosis Detection kit; Roche Diagnostics, Indianapolis, IN, USA). Briefly, the tissue sections were washed in Tris-buffered saline (TBS) and permeabilized using Proteinase K $(20 \mu \mathrm{g} / \mathrm{ml})$ for $10 \mathrm{~min}$. Following permeabilization, the sections were quenched for $5 \mathrm{~min}$ in $3 \% \mathrm{H}_{2} \mathrm{O}_{2}$ in methanol at room temperature (RT). The sections were then incubated in equilibration buffer for $20 \mathrm{~min}$ prior to labeling for $100 \mathrm{~min}$ at $37^{\circ} \mathrm{C}$. The reaction was terminated by stop buffer. Subsequent to further washing in TBS, the sections were incubated in peroxidase-streptavidin conjugate (In Situ Apoptosis Detection Kit) for $45 \mathrm{~min}$, and reacted with 3,3'-diaminobenzidine tetrahydrochloride solution for $15 \mathrm{~min}$ at RT.

Isolation of cortical neurons. Cerebral cortices were isolated and the meninges removed, after which the tissue was minced and treated with $0.25 \%$ trypsin in Earle's balanced salt solution for $1 \mathrm{~min}$. After centrifugation, cortical neurons were isolated. Neurons were isolated from each group, including the sham, saline, $50 \mathrm{ng}$ IL-6 and $500 \mathrm{ng}$ IL-6. Rats were treated with IL-6 (50 ng, i.p.) and IL-6 (500 ng, i.p.), IL-6 solution or a vehicle of physiological saline was administered $10 \mathrm{~min}$ after the MCAO procedure.

Detection of annexin $V$ staining and caspase-3 expression. Cortical neurons, which were prepared and treated as described above, were double-labeled with phycoerythrin (PE)-conjugated caspase-3 monoclonal antibody and fluorescein isothiocyanate (FITC)-conjugated annexin V (BD Pharmingen, San Diego, CA, USA) for $1 \mathrm{~h}$ at RT. PE- and FITC-conjugated murine immunoglobulin $\mathrm{G}$ were used as controls. Subsequent to staining, the cells were assessed using flow cytometry. Cells were fixed and permeabilized, then $5 \times 10^{5}$ cells were stained with $1 \mu \mathrm{g} / \mathrm{ml}$ antibodies against the active form of caspase- 3 and annexin V (BD-Pharmingen) for $60 \mathrm{~min}$ at room temperature. Cell were subsequently washed with phosphate-buffered saline and analyzed in a FACScan flow cytometer (FACSCalibur) and CellQuest software (BD Biosciences, Franklin Lakes, NJ, USA).

Western blot analysis. Protein expression and phosphorylation were detected by western blot analysis. After $12 \mathrm{~h}$ of culturing, the cells were lysed in buffer containing $125 \mathrm{mM}$ Tris- $\mathrm{HCl}$ (pH 6.8), 20\% glycerol, 1\% 2-mercaptoethanol and 2\% sodium dodecyl sulfate (SDS). The total protein from each sample was separated on a $12 \%$ SDS-polyacrylamide gel and electroblotted onto a Hybond-C nitrocellulose membrane (Amersham Pharmacia, Freiburg, Germany). The membrane was subsequently blocked with 5\% non-fat dry milk powder in TBS and incubated for $1 \mathrm{~h}$ with rat monoclonal phospho-Stat1 (\#8826), Stat3 (\#9139) and phospho-Stat3 (\#9145) and polyclonal Stat1 (\#9172) and Bcl2 (\#2876) primary antibodies (1:1,000; Cell 
Signaling Technology, Inc., Danvers, MA, USA). In addition, a rat polyclonal Mcl1 primary antibody (\#A1832) from Selleck Chemicals was used (1:500, Shanghai, China). Following incubation, the membrane was washed four times with $0.05 \%$ Tween-20 in TBS and incubated with peroxidase-conjugated anti-rabbit (\#7074) and anti-mouse (\#7076) IgG secondary antibodies (Cell Signaling Technology, Inc.) for $1 \mathrm{~h}$. The membrane was then washed extensively and the bands in the membrane were developed using enhanced chemiluminescence staining (Amersham Pharmacia).

Measurement of myeloperoxidase (MPO) activity. The enzymatic activity of MPO was measured as an indicator of the accumulation of granulocytes in the ischemic brain tissue (22). Briefly, the brains were rapidly removed at different time-points after MCAO (STAT1/3 and p-STAT1/3, 15 min; Mcl-1 and Bcl-2, 4 h). Samples of ischemic brain tissue weighing $100 \mathrm{mg}$ were isolated, homogenized and centrifuged for $15 \mathrm{~min}$ at $12,000 \times \mathrm{g}\left(4^{\circ} \mathrm{C}\right)$ for later biochemical analysis. An MPO activity assay was conducted using a commercial kit according to the manufacturer's instructions (Nanjing Jiancheng Bioengineering Institute, Nanjing, China) (23). Alterations in the absorbance at $460 \mathrm{~nm}$ were measured using a spectrophotometer (GE Healthcare Bio-Sciences, Pittsburgh, PA, USA). One unit of MPO activity was defined as the degradation of $1 \mathrm{ml} \mathrm{H} \mathrm{O}_{2} \mathrm{O}_{2}$ per min at $37^{\circ} \mathrm{C}$. The final results were expressed as units of MPO activity per gram of wet brain tissue.

Cytokine content measurement in the tissue. Cytokine levels of TNF- $\alpha$, IL-1 $\beta$ and matrix metalloproteinase (MMP)-9 in the rat brain were measured using ELISA kits, according to the manufacturer's instructions (R\&D Systems, Inc., Minneapolis, MN, USA).

Evans blue (EB) leakage. Blood-brain barrier (BBB) permeability was detected by measuring the EB extravasation. EB leakage measurement was performed as described previously (24). The quantity of EB in the supernatant was measured spectrophotometrically at a wavelength of $610 \mathrm{~nm}$ and compared with readings obtained from standard solutions.

Statistical analysis. Quantitative data are presented as the mean \pm standard deviation of at least three independent experiments. Histological injury scoring data were analyzed by analysis of variance (ANOVA) followed by the Kruskal-Wallis nonparametric test for comparison, which is presented as a box-and-whisker plot. The remaining data were analyzed by ANOVA and the Newman-Keuls test for comparison. For comparisons among the groups, the unpaired Student's t-test was performed using GraphPad Prism software (GraphPad Software, Inc., San Diego, CA, USA), in which $\mathrm{P}<0.05$ was considered to indicate a statistically significant difference.

\section{Results}

IL-6 treatment reduces infarct volume and neurological score. To evaluate the efficacy of IL-6 in the rat model of cerebral ischemia/reperfusion, the infarct volume and neurological score were measured $24 \mathrm{~h}$ after the MCAO procedure. In the IL-6 treated rats, the infarct volume (Fig. 1A) and neurological
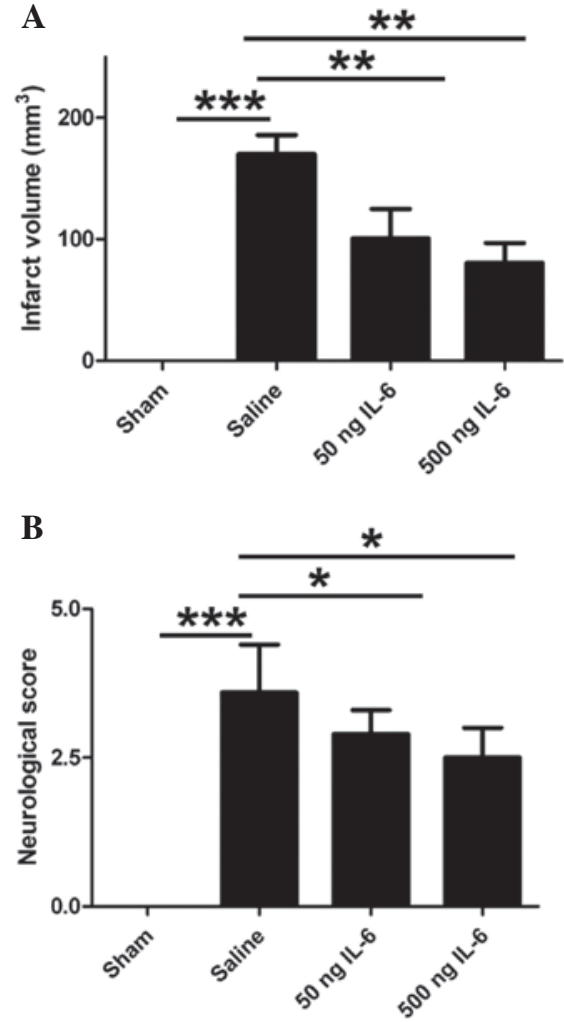

Figure 1. Neuroprotective effect of IL-6 against cerebral ischemia. After $2 \mathrm{~h}$ middle cerebral artery occlusion and $24 \mathrm{~h}$ reperfusion, (A) infarct volume and (B) neurological score were measured in the vehicle (saline)or IL-6 (50 or $500 \mathrm{ng}$ )-treated and sham rats. Results are presented as the mean \pm standard deviation and were assessed using one-way analysis of variance. ${ }^{*} \mathrm{P}<0.05{ }^{* *} \mathrm{P}<0.01$ and ${ }^{* * *} \mathrm{P}<0.001(\mathrm{n}=8)$. IL-6, interleukin-6.

score (Fig. 1B) were reduced significantly in a dose-dependent manner compared with those in the vehicle-treated rats. These results indicate that IL-6 is able to mitigate the damage associated with ischemia/reperfusion-induced brain injury.

IL-6 treatment effectively inhibits ischemia-induced apoptosis. To elucidate the mechanism underlying the neuroprotective effect of IL-6 on ischemia/reperfusion, neuronal apoptosis was determined using TUNEL staining. After $24 \mathrm{~h}$ of reperfusion, MCAO induced considerable DNA fragmentation and a large number of TUNEL-positive cells in the vehicle group compared with the sham group; however, the TUNEL-positive cell count was significantly reduced by the IL- 6 treatment (Fig. 2A). To further confirm the effect of IL-6 on neuronal apoptosis, pure cortical neurons were isolated and the in vivo regulation of apoptosis by IL-6 injection was assessed. Consistently, 50 and $500 \mathrm{ng}$ doses of IL-6 effectively inhibited the ischemia-induced apoptosis, as indicated by annexin $\mathrm{V}$ binding, compared with the vehicle control (Fig. 2B). In addition, the number of activated caspase-3-positive neurons also increased markedly in the vehicle group compared with the sham group (Fig. 2C), while IL-6 treatment attenuated this increase (Fig. 2C). These results indicate that IL-6 mitigates ischemia-induced neuronal apoptosis.

IL-6 modulates neuronal anti-apoptotic proteins by activating STAT3 in the JAK/STAT pathway. To further elucidate the 

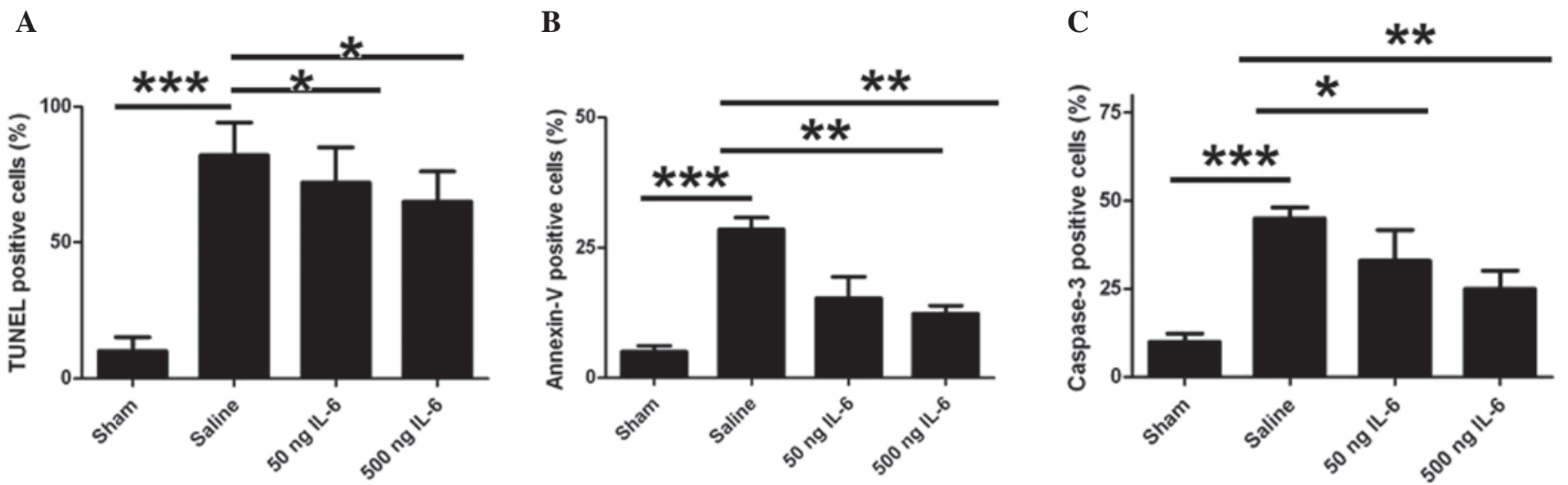

Figure 2. Effect of IL-6 on neuronal apoptosis in ischemia/reperfusion. (A) Qualification of TUNEL-positive cells. Levels of neuronal apoptosis and DNA fragmentation in tissue sections were determined by TUNEL staining. The number of TUNEL-positive cells in the sham, saline- and IL-6-treated rats was counted, respectively. (B) Cortical neurons isolated from each group were stained with fluorescein isothiocyanate-labeled annexin V to detect neuronal apoptosis. Cells were analyzed by FACS and the histogram shows the representative percentage of annexin V-positive cells. (C) The number of caspase-3-positive apoptotic cells was calculated in cortical neurons isolated from each group using FACS; the representative percentage of caspase-3-positive cells is presented in the histogram. ${ }^{*} \mathrm{P}<0.05,{ }^{* *} \mathrm{P}<0.01$ and ${ }^{* * *} \mathrm{P}<0.001$. IL-6, interleukin-6; TUNEL, terminal deoxynucleotidyl transferase-mediated dUTP nick end labeling; FACS, fluorescence-activated cell sorting.

A

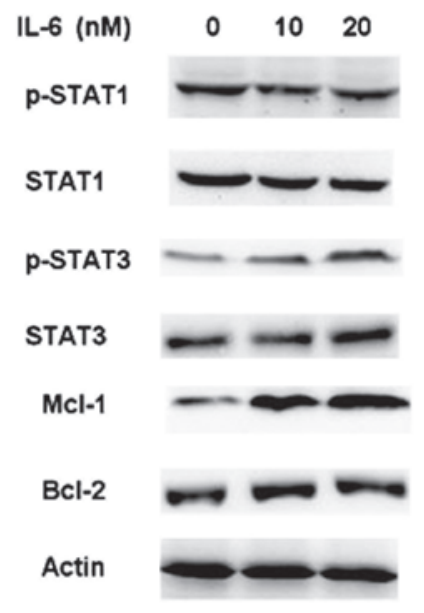

C

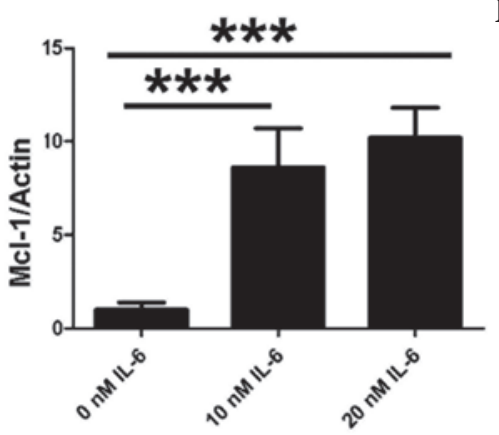

B

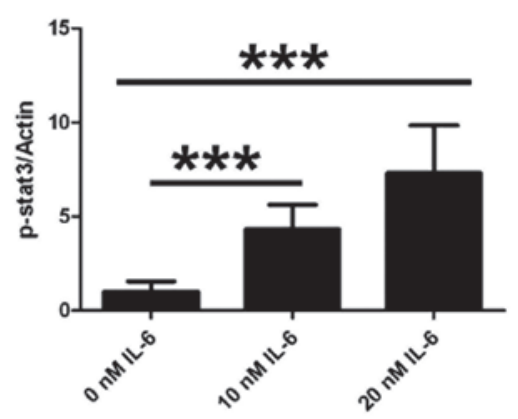

D

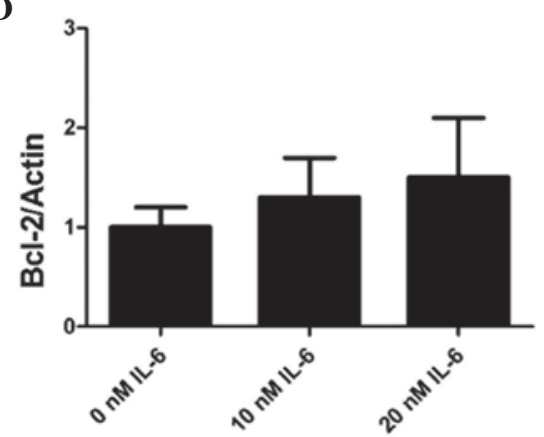

Figure 3. Putative pathway involved in the IL-6-mediated inhibition of apoptosis in the ischemic brain. (A) Cortical neurons were isolated from vehicle-treated rats. Following exposure to the indicated concentrations of IL-6, the neurons were subjected to western blot analysis of various proteins at different time-points: STAT1/3 and p-STAT1/3, 15 min; Mcl-1 and Bcl-2, $4 \mathrm{~h}$. The membrane was first hybridized with anti-phospho-STAT1/3 antibody, and then rehybridized with an anti-STAT1/3 antibody. $\beta$-actin was used as a control for protein loading. (B-D) Statistical data from the western blot analysis for the phosphorylation or protein expression of STAT3, Mcl-1 and Bcl-2. ${ }^{* * *} \mathrm{P}<0.001$. IL-6, interleukin-6; STAT, signal transducer and activator of transcription; Mcl-1, induced myeloid leukemia cell differentiation protein Mcl-1; Bcl-2, B-cell lymphoma 2.

mechanism underlying the inhibitory function of IL-6 on apoptosis, apoptotic proteins were examined using western blot analysis. To specifically investigate the JAK/STAT pathway, the cells were treated with various concentrations of IL-6 in vitro.
As shown in Fig. 3A and B, IL-6 induced the phosphorylation of STAT3 while exerting no effect on total STAT3 expression. No difference was observed in the phosphorylation of STAT1 and total STAT1 expression following IL-6 treatment under 

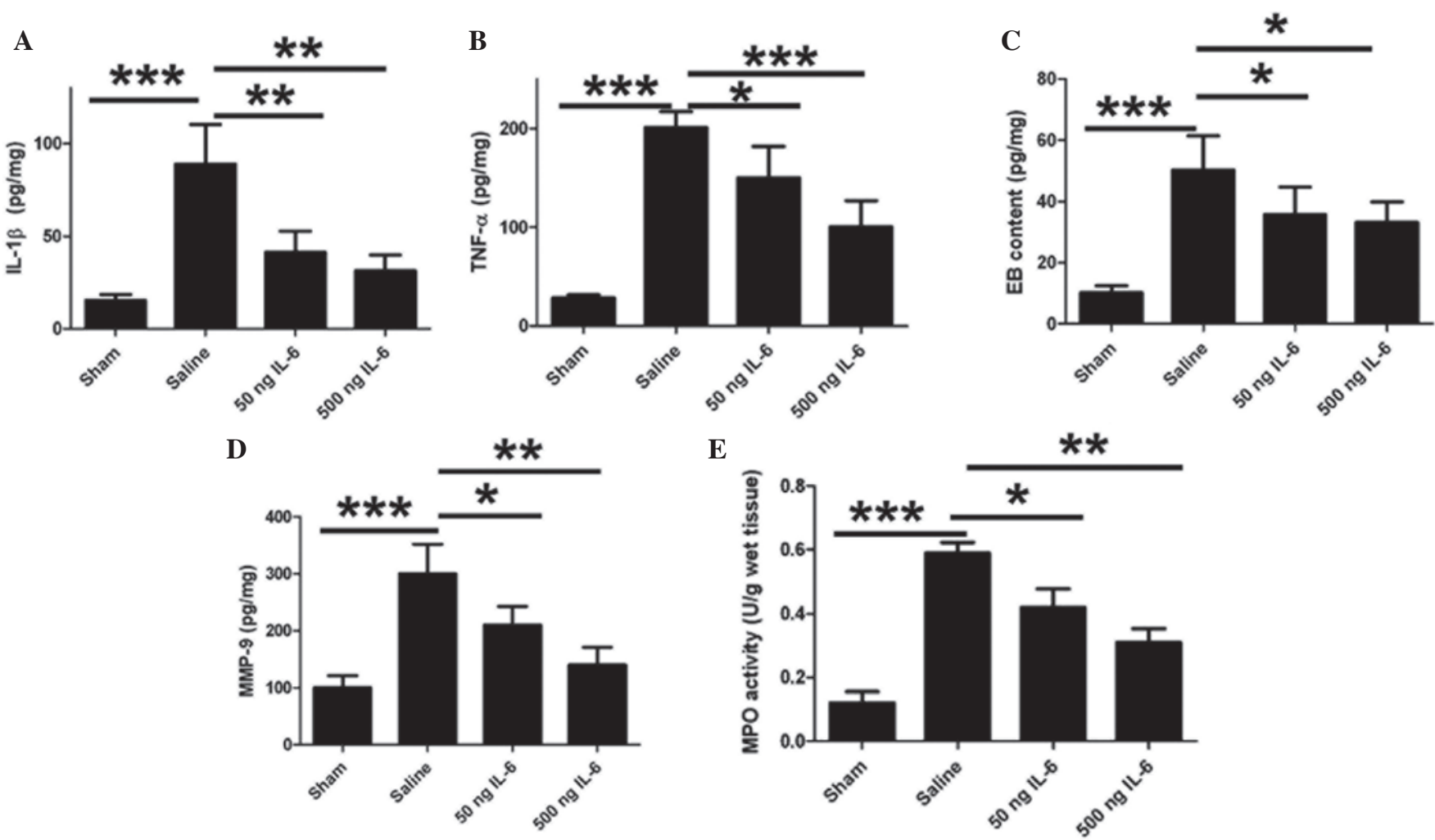

Figure 4. Effect of IL-6 on the central nervous system inflammatory response and blood-brain barrier permeability in rats following cerebral ischemic injury. (A-E) Effect of IL-6 on (A and B) the inflammatory IL-1 $\beta$ and TNF- $\alpha$ levels (determined by ELISA); (C) the EB content in rats brain following ischemia-reperfusion; (D) MMP-9 levels (determined by ELISA); and (E) the enzymatic activity of MPO. The ischemic brain was analyzed 24 h after middle cerebral artery occlusion. ${ }^{*} \mathrm{P}<0.05,{ }^{* *} \mathrm{P}<0.01$ and ${ }^{* * *} \mathrm{P}<0.001$. IL, interleukin; TNF- $\alpha$, tumor necrosis factor- $\alpha$; EB, Evans blue; MMP-9, matrix metalloproteinase-9; MPO, myeloperoxidase.

identical experimental conditions (Fig. 3A). Furthermore, neuronal Mcl-1 expression was upregulated following the IL-6 treatment (Fig. 3C), but the treatment did not result in any difference in Bcl-2 expression (Fig. 3D). These results suggest that IL-6 modulates neuronal anti-apoptotic proteins by activating STAT3 in the JAK/STAT pathway.

$I L-6$ treatment reduces levels of $I L-1 \beta$ and TNF- $\alpha$ and ameliorates $E B$ leakage. The levels of a number of inflammatory cytokines were quantified in order to determine whether IL-6 influences the regulation of cytokine secretion and BBB integrity in cerebral ischemia As shown in Fig. 4A and B, IL-6 induced a significant reduction in the levels of IL- $1 \beta$ and TNF- $\alpha$ in the rat brain, suggesting that IL-6 mediates the immune response following the inhibition of neuronal apoptosis. As inflammatory cytokines are responsible for the BBB integrity, the effect of IL-6 on the BBB permeability following ischemia/reperfusion was subsequently investigated. EB extravasation was detected in the ischemic region; however, IL-6 injection notably reduced this EB leakage in vivo (Fig. 4C). High levels of MMP-9 expression were observed in the ischemic brain tissue, and IL-6 reduced these levels (Fig. 4D). Furthermore, IL-6 reduced MPO activity in a dose-dependent manner (Fig. 4E).

\section{Discussion}

Apoptosis is a typical cell function with various characteristic morphological features, including DNA fragmentation,

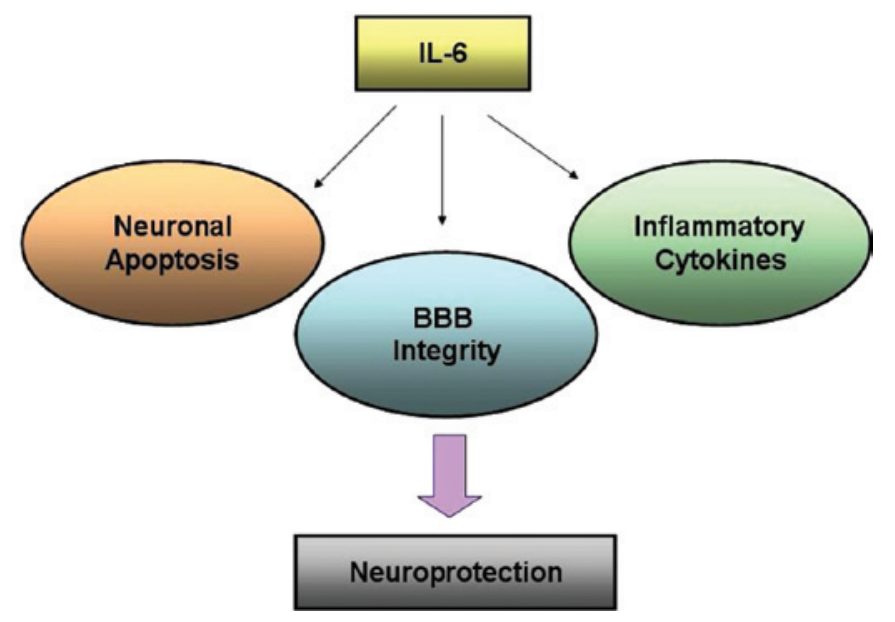

Figure 5. Putative mechanism of IL-6 in cerebral ischemia. The effect of IL-6 on cerebral ischemia is attributed to the IL-6-mediated neuronal apoptosis decrease in vivo and in vitro. IL-6 regulates the apoptosis-related signaling in the neuron, inhibits the inflammatory cytokines in the brain and maintains the integrity of the BBB in the injured tissue, suggesting that IL-6 plays an important role in the therapy of cerebral ischemia-related neuronal diseases. IL-6, interleukin-6. BBB, blood-brain barrier.

nuclear chromatin condensation and cell shrinkage. Apoptosis is widely implicated in neuronal disease; for example, apoptosis can lead to neuronal death in Alzheimer's disease, and amyloid $\beta$-protein may be involved in this process $(25,26)$. It is, however, unknown which specific factors regulate the 
apoptosis of neurons following cerebral ischemia. To confirm the anti-apoptosis effect of IL-6, the rate of neuronal apoptosis was measured using a number of independent methods in the present study. A TUNEL assay demonstrated that cerebral ischemia induced DNA fragmentation. Consistently, IL-6 also reduced annexin $\mathrm{V}$ binding and caspase- 3 expression in freshly isolated cortical neurons compared with the cells from the saline-treated group. These results therefore demonstrate that IL-6 protects neurons against apoptosis. To elucidate the possible associated mechanism, the signal transduction was investigated in neurons from cerebral ischemia mice in vitro.

In the present study, high expression levels of Mcl-1 were observed to be associated with reduced levels of apoptosis in the IL-6-treated injured neurons. Mcl-1 is implicated in myeloid pathways upon exposure to 12-O-tetradecanoylphorbol-13-acetate (27). Although Mcl-1 has a key function in B-cell differentiation and survival, the exact of role of Mcl-1 has not been defined $(28,29)$. The results of the present study additionally reveal that IL-6 induces STAT3 phosphorylation in primary neuronal cells. We therefore hypothesized that the phosphorylation of STAT3 in the JAK/STAT pathway stimulates Mcl-1 expression. Consequently, these results suggest that STAT3 is involved in the IL-6-mediated anti-apoptosis activity, and the JAK/STAT pathway $(30,31)$ may serve a key function in mediating Mcl-1 and the apoptotic processes.

The present study indicated that IL-6 is able to alleviate the cerebral ischemic/reperfusion damage in a rat model. Furthermore, IL-6 exerted a neuroprotective effect by inhibiting neuronal apoptosis and inflammatory mediators in the brain. Increased MPO activity was observed following the ischemic injury; however, IL-6 reduced the MPO activity, suggesting that IL-6 is able to inhibit the inflammatory responses in the brain. This observation was confirmed by the downregulation of inflammatory cytokines, including TNF- $\alpha$ and IL-1 $\beta$. These results indicate that IL- 6 ameliorates the symptoms of ischemic brain injury by preventing the secretion of inflammatory cytokines and the immune response in the brain. The breakdown of the BBB is involved in the pathogenesis of cerebral ischemia, and the present data regarding the reduced MMP-9 expression following IL-6 treatment are consistent with the reduction in EB content in the rat brain $(32,33)$. This therefore suggests that IL- 6 protects the $\mathrm{BBB}$ and exerts a neuroprotective effect in MCAO-induced cerebral ischemia. To the best of our knowledge, the present study is the first to demonstrate the efficacy of an inflammatory cytokine in a rat model of cerebral ischemia in rats via anti-inflammation and anti-apoptosis pathways (Fig. 5).

In conclusion, the results of the present study suggest that IL-6 plays a comprehensive role in cerebral ischemia by mediating neuronal apoptosis, inflammatory cytokines and BBB integrity in the CNS (Fig. 5). The present study has thus elucidated a possible mechanism underlying the actions of IL-6 in this disease and has indicated the possibility of the application of IL- 6 as a therapeutic agent for cerebral ischemia.

\section{Acknowledgements}

The study was supported by a grant from the Natural Science Foundation of Hubei Province, China (no. 070413066).

\section{References}

1. Amantea D, Nappi G, Bernardi G, Bagetta G and Corasaniti MT: Post-ischemic brain damage: Pathophysiology and role of inflammatory mediators. FEBS J 276: 13-26, 2009.

2. Wang Y, Cao M, Liu A, Di W, Zhao F, Tian Y and Jia J: Changes of inflammatory cytokines and neurotrophins emphasized their roles in hypoxic-ischemic brain damage. Int J Neurosci 123: 191-195, 2013.

3. Pradillo JM, Denes A, Greenhalgh AD, Boutin H, Drake C, McColl BW, Barton E, Proctor SD, Russell JC, Rothwell NJ and Allan SM: Delayed administration of interleukin-1 receptor antagonist reduces ischemic brain damage and inflammation in comorbid rats. J Cereb Blood Flow Metab 32: 1810-1819, 2012.

4. Kishimoto T: The biology of interleukin-6. Blood 74: 1-10, 1989.

5. Wakabayashi K, Nagai A, Sheikh AM, Shiota Y, Narantuya D, Watanabe T, Masuda J, Kobayashi S, Kim SU and Yamaguchi S: Transplantation of human mesenchymal stem cells promotes functional improvement and increased expression of neurotrophic factors in a rat focal cerebral ischemia model. J Neurosci Res 88: 1017-1025, 2010.

6. Nakamachi T, Tsuchida M, Kagami N, Yofu S, Wada Y, Hori M, Tsuchikawa D, Yoshikawa A, Imai N, Nakamura K, et al: IL-6 and PACAP receptor expression and localization after global brain ischemia in mice. J Mol Neurosci 48: 518-525, 2012

7. Hama T, Kushima Y, Miyamoto M, Kubota M, Takei $N$ and Hatanaka H: Interleukin-6 improves the survival of mesencephalic catecholaminergic and septal cholinergic neurons from postnatal, two-week-old rats in cultures. Neuroscience 40: 445-452, 1991.

8. Dooley D, Vidal P and Hendrix S: Immunopharmacological intervention for successful neural stem cell therapy: New perspectives in CNS neurogenesis and repair. Pharmacol Ther 141: 21-31, 2014.

9. Brázda V, Klusáková I, Hradilová Svíženská I and Dubový P: Dynamic response to peripheral nerve injury detected by in situ hybridization of IL-6 and its receptor mRNAs in the dorsal root ganglia is not strictly correlated with signs of neuropathic pain. Mol Pain 9: 42, 2013.

10. Allodi I, Udina E and Navarro X: Specificity of peripheral nerve regeneration: Interactions at the axon level. Prog Neurobiol 98: 16-37, 2012.

11. Hibi M, Nakajima K and Hirano T: IL-6 cytokine family and signal transduction: A model of the cytokine system. J Mol Med Berl 74: 1-12, 1996.

12. Heim MH: The Jak-STAT pathway: Cytokine signalling from the receptor to the nucleus. J Recept Signal Transduct Res 19: 75-120, 1999.

13. Heinrich PC, Behrmann I, Müller-Newen G, Schaper F and Graeve L: Interleukin-6-type cytokine signalling through the gp130/Jak/STAT pathway. Biochem J 334: 297-314, 1998.

14. Adams JM and Cory S: The Bcl-2 protein family: Arbiters of cell survival. Science 281: 1322-1326, 1998.

15. Puthier D, Pellat-Deceunynck C, Barillé S, Robillard N, Rapp MJ, Juge-Morineau N, Harousseau JL, Bataille R and Amiot M: Differential expression of Bcl-2 in human plasma cell disorders according to proliferation status and malignancy. Leukemia 13: 289-294, 1999.

16. Tu Y, Renner S, Xu F, Fleishman A, Taylor J, Weisz J, Vescio R, Rettig M, Berenson J, Krajewski S, et al: BCL-X expression in multiple myeloma: Possible indicator of chemoresistance. Cancer Res 58: 256-262, 1998.

17. Altmeyer A, Simmons RC, Krajewski S, Reed JC, Bornkamm GW and Chen-Kiang S: Reversal of EBV immortalization precedes apoptosis in IL-6-induced human B cell terminal differentiation. Immunity 7: 667-677, 1997.

18. Lømo J, Smeland EB, Krajewski S, Reed JC and Blomhoff HK: Expression of the Bcl-2 homologue Mcl-1 correlates with survival of peripheral blood B lymphocytes. Cancer Res 56: 40-43, 1996.

19. Mérino D, Khaw SL, Glaser SP, Anderson DJ, Belmont LD, Wong C, Yue P, Robati M, Phipson B, Fairlie WD, et al: Bcl-2, $\mathrm{Bcl}-\mathrm{x}(\mathrm{L})$, and Bcl-w are not equivalent targets of ABT-737 and navitoclax (ABT-263) in lymphoid and leukemic cells. Blood 119: 5807-5816, 2012.

20. Dimberg LY, Dimberg A, Ivarsson K, Fryknäs M, Rickardson L, Tobin G, Ekman S, Larsson R, Gullberg U, Nilsson K, et al: Stat1 activation attenuates IL-6 induced Stat 3 activity but does not alter apoptosis sensitivity in multiple myeloma. BMC Cancer 12: 318,2012 . 
21. Longa EZ, Weinstein PR, Carlson S and Cummins R: Reversible middle cerebral artery occlusion without craniectomy in rats. Stroke 20: 84-91, 1989.

22. Matsuo Y,Onodera H,Shiga Y,NakamuraM,NinomiyaM,Kihara T and Kogure K: Correlation between myeloperoxidase-quantified neutrophil accumulation and ischemic brain injury in the rat. Effects of neutrophil depletion. Stroke 25: 1469-1475, 1994.

23. Lou HY, Zhang XM, Wei XB, Wang RX and Sun X: Anti-inflammatory effect of hydroxyethylpuerarin on focal brain ischemia/reperfusion injury in rats. Chin J Physiol 47: 197-201, 2004.

24. Gürsoy-Ozdemir Y, Bolay H, Saribaş O and Dalkara T: Role of endothelial nitric oxide generation and peroxynitrite formation in reperfusion injury after focal cerebral ischemia. Stroke 31: 1974-1981, 2000.

25. LaFerla FM, Tinkle BT, Bieberich CJ, Haudenschild CC and Jay G: The Alzheimer's A beta peptide induces neurodegeneration and apoptotic cell death in transgenic mice. Nat Genet 9: 21-30, 1995.

26. Miravalle L, Tokuda T, Chiarle R, Giaccone G, Bugiani O, Tagliavini F, Frangione B and Ghiso J: Substitutions at codon 22 of Alzheimer's abeta peptide induce diverse conformational changes and apoptotic effects in human cerebral endothelial cells. J Biol Chem 275: 27110-27116, 2000.

27. Yang T, Buchan HL, Townsend KJ and Craig RW: MCL-1, a member of the BLC-2 family, is induced rapidly in response to signals for cell differentiation or death, but not to signals for cell proliferation. J Cell Physiol 166: 523-536, 1996.
28. Zhou P, Qian L, Bieszczad CK, Noelle R, Binder M, Levy NB and Craig RW: Mcl-1 in transgenic mice promotes survival in a spectrum of hematopoietic cell types and immortalization in the myeloid lineage. Blood 92: 3226-3239, 1998.

29. Reynolds JE, Yang T, Qian L, Jenkinson JD, Zhou P, Eastman A and Craig RW: Mcl-1, a member of the Bcl-2 family, delays apoptosis induced by c-Myc overexpression in Chinese hamster ovary cells. Cancer Res 54: 6348-6352, 1994.

30. Jin S, Mutvei AP, Chivukula IV, Andersson ER, Ramsköld D, Sandberg R, Lee KL, Kronqvist P, Mamaeva V, Ostling P, et al: Non-canonical Notch signaling activates IL-6/JAK/STAT signaling in breast tumor cells and is controlled by p53 and IKK $\alpha /$ IKK $\beta$. Oncogene 32: 4892-4902, 2013.

31. Fahmi A, Smart N, Punn A, Jabr R, Marber M and Heads R: p42/p44-MAPK and PI3K are sufficient for IL-6 family cytokines/gp130 to signal to hypertrophy and survival in cardiomyocytes in the absence of JAK/STAT activation. Cell Signal 25: 898-909, 2013.

32. Zhang X, Zhang X, Wang C, Li Y, Dong L, Cui L, Wang L, Liu Z, Qiao H, Zhu C, et al: Neuroprotection of early and short-time applying berberine in the acute phase of cerebral ischemia: Up-regulated pAkt, pGSK and pCREB, down-regulated NF- $\kappa B$ expression, ameliorated BBB permeability. Brain Res 1459: $61-70,2012$.

33. Nagaraja TN, Keenan KA, Brown SL, Fenstermacher JD and Knight RA: Relative distribution of plasma flow markers and red blood cells across BBB openings in acute cerebral ischemia. Neurol Res 29: 78-80, 2007. 